\title{
Experiment in Non Word Repetition by Monolingual Arabic Preschoolers
}

\begin{abstract}
By Amani Jaber-Awida*
The paper attempted to check children's phonological memory and phonological awareness by asking them to repeat various non-words. A non word repetition experiment was conducted, using 30 non words which ranged from 2 to 4 syllables. Half of the non words were target-like and half were non target-like. Subjects were asked to repeat the non words as accurately as they could. Results showed that long non words were repeated more erroneously than short non words. In addition, word-likeness influenced the accuracy of repetition; where non words with high word likeness taxed more errors by children. A note was provided on specific items and individuals which triggered more errors than others.
\end{abstract}

Keywords: non-word repetition, monolinguals, phonology, short-term memory, vocabulary acquisition

\section{Background}

Non Word Repetition (i.e. NWR) is a method by which subjects are asked to repeat words not existing in the language in order to test their phonological short term memory. Gathercole defined Non Word Repetition in 1989 as a measure of phonological short-term memory, yet redefined it in 2006, as the ability to store phonological items which essential in non-word repetition and vocabulary acquisition (Chiat 2006).

Working memory is strongly related to language processing. It is divided into the following subsystems: the phonological loop, the visuospatial sketchpad, the central executive, and recently the episodic buffer. It is crucial to clarify what each subsystem is responsible for in order to understand the vitality of having then as functioning subsystems. First, the phonological loop deals with phonological information and it is necessary for the immediate retention of digits. According to the phonological loop, it is easier to remember a sequence of dissimilar words than similar ones. The phonological loop also contributes to the acquisition of first and second languages. The visuospatial sketchpad, on the other hand, is a visual subsystem and is less relevant for language, but it helps in comprehension. The central executive is responsible for the attentional control of working memory. And lastly, the episodic buffer can bind together information from different sources into chunks. If any of these subsystems malfunctions, there will be a serious problem in language processing (Baddeley 2003).

Chiat (2006) stated that in the first year of children's lives they are equipped with sensitivity to the "properties and frequency of rhythmic patterns in phonological input" and they utilize this sensitivity to segmenting words for storage. According to this hypothesis, it is claimed that children will vary in their ability to repeat non words because they will differ in their early phonological

${ }^{*} \mathrm{PhD}$ Student, Bar Ilan University, Israel. 
sensitivity. Vocabulary growth is said to be a catalyst for non word repetition ability. It supports non word repetition ability and it undergoes change with age (Chiat 2006). Findings by Gathercole (2006) show that SLI children are more impaired on non word repetition tasks than serial recall tasks, even when the stimuli on both tasks has identical syllables. Another finding by Gathercole was that some children with poor ability of non word repetition and serial recall at age 5 have age appropriate linguistic abilities at age 8. These findings led Gathercole to assume that a phonological storage deficit is not solely what disrupts language development, but working memory might play a role as well. In addition, it was also inferred that non word repetition might be a very "effective predictor of language learning ability" at some stages of the trajectory of language development (Chiat 2006).

Generally, non word repetition tasks were used to identify SLI children among bilingual populations. For instance, Girbau and Schwartz (2008) examined the phonological working memory, i.e. "the process of receiving, analyzing and processing of sound elements in language" of Spanish-English bilinguals aging 8-10 years old with and without SLI in order to pin point the differences children have performing the task and consequently identify them as SLI or typically developing children (TD). Additionally, the researchers conducted a pilot study and tested the mothers of the children to check if the mothers of SLI'ers will do more poorly on the NWR task than the TD mothers. The study included 20 non words with five syllable lengths (1-5) following the Spanish phonotactic patterns. Results showed that most errors occurred in repeating non words with the length of 3 and more syllables. This length effect can perhaps serve as an answer to the sub-question in the research question section of this paper. Vowels were rarely repeated erroneously, yet SLI children tended to err more on consonants especially with long non words. The most prevalent type of error was consonant substitution for both groups, though SLI erred more in this type of mistake and also on mistakes of consonant omission and clusters. Both groups produced non words with less frequent segment sequence poorer than those that are more frequent. This suggests that it is not merely a matter of length of utterance, but there are additional factors playing a role in the repetition performance of children, such as frequency.

Furthermore, the mothers of SLI children were poorer than TD mothers at the NWR task. This study revealed that phonological working memory abilities are related to the comprehension and production of children in their native language, i.e. Spanish (Girbau \& Schwartz 2008). The similarity of performance by SLI mothers and their children suggests, in my opinion, the question: does heredity play a role in this case? Or does the fact that SLI children perform in a certain way influence their mothers' performance due to their shared environment and linguistic interaction?

Gray (2003) conducted a study in which she aimed to check the reliability and accuracy of non word repetition tasks and digit span tasks used for SLI preschool children. The study aimed to check if non word repetition was a useful diagnostic tool for previously studied children. For this purpose, Gray had three groups of subjects: SLI, age matched normally developing children, 
and gender matched normally developing children; with the age range of 4 and 6. The study used two forms of the CNRep test, where the lists were randomly divided by syllable length. Children were given time to practice the tests lists, and the tests were repeatedly conducted with a fixed time between each trial. Results of the study showed that the SLI group and the normally developing groups' performances increased from the first test trial to the second, yet, it decreased from the second trial to the second. Gray indicates that the resulting scores show a clear discrimination between the SLI and the normal developing children. Gray relates to the results of the first and second trials of the tests as well as the second and third trials with doubting the reliability of these tests. She explains her doubts by claiming that the results might be due to practice the children had. Thus, Gray concludes that further research is needed before determining that non word repetition tests are fully reliable to be used as diagnostic tools for SLI (Gray 2003).

SLI children struggle to a greater extent in repeating non words like loddernapish than TD children (Bishop et al. 1996, Edwards \& Lahey 1998, Ellis Weismer et al. 2000, Gathercole \& Baddeley 1990, Montgomery 1995). It is suggested by many researchers that word likeness influences the performance of children in repeating non words. Children find it easier to repeat non words with high word likeness than non words with low word likeness (Coady et al. 2005, Gathercole 1995, Munson et al. 2005). Graf Estes et al. (2007) examined unpublished data in order to check the performance of children with and without SLI on non word repetition tasks. They found that SLI children performed worse than their peers without SLI in repeating long and short non words. However, SLI children had a greater difficulty in repeating long non words. In Graf Estes et al. (2007) it is further explained that SLI children's difficulties may result from the fact that they might be impaired at a point or several points of the following process: when a children hears a new phonological sequence he/she mush encode the sequence and hold it temporarily in memory and after undergoing a processing for articulation, the sequence is connected to meaning. Generally they concluded that, "non word length, articulatory complexity, and wordlikeness" negatively affect the performance of SLI children in repeating non words (Graf Estes et al. 2007). This, in turn, is reflected in their processing capacities compared to TD children.

Phonological impairment might interact with grammatical impairment the child might have (Van der Lely et al. 2007). SLI children omit consonant clusters when repeating non words and have difficulty dealing with weak syllables. Van der Lely et al. (2007) claim that this is a result of a deficit in the short term working memory and phonological processing. Yet, in 1993 van der Lely and Howard claimed that these difficulties might be also a result of the prosodic structure non words might have. Van der Lely et al. (2007) conducted a study to investigate the phonological abilities of English speakers with Grammatical SLI. The study included 13 G-SLI children and 24 TD children with mean age 15.96 non words were included which varied in their complexity. This study revealed that the performance of G-SLI children decreases as syllable length increases, and when the number of prosodic structures increases the G-SLI children performance 
decreases also. Mapping vowels and consonants onto target prosodic structure is an obstacle for G-SLI children. Moreover, short term memory contributes to the repetition accuracy of children. On the other hand, prosodic complexity affects the performance of G-SLI children and implies a deficit in their phonological grammar (Van der Lely et al. 2007).

Archibald and Gathercole (2006) compared the performance of SLI children with two different groups of children, who were matched for age and language ability, on two tests of non word repetition. The children's age range was 7 to 11 years old. The two non word repetition tests were Children's Test of Nonword Repetition (CNRep) and the Nonword Repetition Test (NRT); meant to investigate what might contribute to the deficit of non word repetition in Specific Language Impairment (SLI) children. Results showed that SLI children were disadvantaged than their age matched peers on both tests. However, SLI children performed poorer only in the CNRep test than their language matched peers. SLI children also had difficulty and lagged behind the other two groups in repeating long non words and non words containing consonant clusters. Archibald and Gathercole explain these differences in performance by claiming that the SLI poor performance may be an outcome of verbal short term memory, a deficit in lexical knowledge or output processes (Archibald \& Gathercole 2006). This is also supported in Sahlén (1999) study in which Sahlén found that "maturity of phonological output processes was strongly associated with non-word repetition scores in a sample of young children with language impairment" (Archibald \& Gathercole 2006). The present study of Archibald and Gathercole (2006) mentions that Conti-Ramsden et al. (2001) utilized the CNRep as a clinical marker for SLI children ages 5 (Conti-Ramsden 2003) and 11 year old children with former SLI (Conti-Ramsden et al. 2001). Thus, lexical knowledge would be a contributor to non word repetition capacity.

Studies show that SLI children generally recall less information because they have general cognitive deficits such as low processing capacity or processing rate. Other explanations are that language impairment is a result of a deficit in the short term memory system which is related to the auditoryverbal information (Balthazar 2003). Balthazar (2003) referred the word length effect as the ability of subjects to recall short and long words. It is claimed for instance that a list of short words like "bat, key, cub, man, duck" is easier to recall than the list of long words like "hamburger, alligator, Halloween, tuition, metaphor". The reason behind this claim that it is more time consuming to subvocally repeat long words than short ones (Balthazar 2003). Various studies indicate that children are not born with the ability to rehearse; rather, they develop this ability from the age of seven on (Cuvo 1975, Kail 1990, Ornstein et al. 1977). They become better at deliberate rehearsals as age develops (Hagen \& Kail 1973, Keeney et al. 1967). In addition,

"...when children are involved, the word length effect can be influenced by factors other than rehearsal, including rate of overt articulation, during the recall phase (Henry 1991b), word identification time (Dempster 1981), cognitive and perceptual capacity (Cohen \& Heath 1990), speed of memory search (Cowan et al. 1994, 
Sininger et al. 1989, and response planning time (Sininger et al. 1989)". (as cited in Balthazar 2003: 490)

Consequently, we can conclude that exposure to regular meaningful language triggers a hidden ability one has for non word repetition.

Balthazar (2003) investigated the "word length effect as a measure of subvocal rehearsal in children with language impairment". Two tasks were used, the full verbal recall task (FR) and the probed recall task (PR). The study examined three groups of subjects: SLI group, age matched group, and expressive language matched group. The lists of words in the tasks were divided into lists of short words and others of long words. The words in each list were not related semantically or phonologically. Results showed that the three groups showed word length effect, however, the impaired group performed worse than their age and language matched peers in recalling lists of long words. The age matched group outperformed the other two groups in recalling greater number of word lists. In summary, the impaired group had similar results in the PR task like the other groups, in contrast to the results in the FR task (Balthazar 2003). Some attribute these differences to the possibility that children with language impairment might be slower in the speed of efficiency of processing (Kail 1994, Lahey et al. 2001, Sininger et al. 1989).

Since SLI identification has been a subject of interest for many linguists and researchers, there have been studies attempting to test the reliability of different tasks as being SLI potential identifiers or markers. Yet, there is a dearth in the number of studies that examine many tasks or measures simultaneously. Among the few studies examining a number of measures at a time is a study by ContiRamsden et al. (2001) who looked at a group of 11 children previously diagnosed with SLI in three tasks: non word repetition, tense marking, and sentence repetition. They concluded that sentence repetition was the most useful marker for SLI children (Conti-Ramsden 2003). Conti-Ramsden (2003) was interested in knowing how to distinguish SLI children from non SLI ones, and for that purpose conducted a study using processing and linguistic markers to check if they were good markers for diagnosing SLI children. The processing markers were: non word repetition and digit recall, whereas the linguistic markers were: past tense and plural marking (Conti-Ramsden 2003). For the purpose of this paper, we will focus on the processing markers. In the non word repetition task Conti-Ramsden used items from the CNRep test included in Gathercole and Baddeley's 1996 experiment. The non words were 2-5 syllables long. Results in the non word repetition tasks showed that SLI children had a mean score of 27.59 while their normal peers had a mean score of 78.34. Overall; the results indicated that SLI children performed worse than their normal peers in all the four markers tested in the study (Conti-Ramsden 2003). This supports previous research claiming that SLI children have difficulties in processing and linguistic tasks (Ellis Weismer et al. 2000, Conti-Ramsden et al. 2001). Conti-Ramsden states that it is still early to decide that the aforementioned markers should be definitely used for clinical purposes, and it is also important to note that further research in other languages is in need (2003). 
Alloway and Gathercole (2005) mention that "memory span for words in sentences is almost twice as great as the span for unrelated sequences of words" (Baddeley et al. 1987, Butterworth et al. 1990). It is widely agreed also that the advantage of recalling words in sentences stems from the contribution of the meaning of the sentence. There are several factors influencing the recall of unrelated words as well as words in sentences. These factors are: high phonological similarity of words negatively affects the comprehension and recall of words (Baddeley 1986). In addition, the longer the words, the less accurate the recall will be (Engelkamp \& Rummer 1999, Rummer \& Engelkamp 2001, Willis \& Gathercole 2001). Moreover, short term memory impairment results in poor recall in word lists and sentences, despite the fact that the comprehension of such individuals is usually intact (Warrington \& McCarthy 1987). Some researchers (Hanten \& Martin 2000, Martin et al. 1999) claim that "phonological and semantic short term memories contribute to immediate memory for sentences" (Alloway \& Gathercole 2005). While Baddeley (2000) maintains that memory of sentences is aided by an episodic buffer, where the "phonological representation of the sentence is combined with the conceptual representations resulting from language processing" (Alloway \& Gathercole 2005). Alloway and Gathercole (2005) investigated the link between phonological memory and short term sentence recall. Their study had children with poor phonological short-term memory skills and others with good phonological short-term memory skills. They used three measures to test the children: digit recall and word recall tests (Gathercole \& Pickering 2000), along with a non word repetition test (Gathercole \& Baddeley 1996). Alloway and Gathercole (2005) found that children with the high phonological memory had accuracy of $82 \%$ in sentence recall; yet, the low phonological memory group scored $52 \%$. Words in initial positions were recalled better than words in middle and final positions generally by both groups. It was also found that the high memory group tended to make more substitution errors (high group $=67 \%$, low group $=45 \%$ ), and the low memory group made more non substitution errors (low group $=55 \%$, high group $=33 \%$ ). In conclusion, both groups substituted target words with synonyms more often; something that suggests that semantic information may contribute to the recall of sentences (Alloway \& Gathercole 2005).

Word length and likeness seem to greatly contribute to the preciseness of repeating non words by language learners. Reviewing the literature of non word repetition showed that non word repetition can indicate how successful language learners will be in learning or acquiring a new language. Below is a review of the word structure of Arabic to reflect on the process Arabic learners undergoing once engaged in non word repetition tasks.

\section{Word Structure in Arabic}

Arabic as a Semitic language has its own unique features. The basic meaningful word root in Arabic contains three consonants, e.g. f.a".l (i.e. Act). If we omit one of its letters, the word becomes meaningless. From this basic word 
root we can build a wide range of vocabulary by affixation, and in turn get to the various conjugations Arabic language has. For example: infaa"la (i.e. got excited), in the given example the tri-consonantal root was prefixed and suffixed at the same time.

In creating my list of non words I used the different conjugations in Arabic and created non words with each conjugation structure. For example: "salba" came from the conjugation "faa"la", "idkaman" came from the conjugation "iftiaal", etc.

\section{Research Question and Hypothesis}

\section{Research Question}

Will Arabic speaking monolingual preschool children succeed to repeat non words which are target like items with the same accuracy they repeat non words with are non target like items?

Will length of items influence the results?

\section{Hypothesis}

1. It will be easier for preschool children to repeat non words which are target like items. This is consistent with the claims made by many researchers that word likeness influences the performance of children in repeating non words. Children find it easier to repeat non words with high word likeness than non words with low word likeness (Coady et al. 2005, Gathercole 1995, Munson et al. 2005). In addition, Girbau and Schwartz (2008) state that frequency influences children's performance on non word repetition tasks, where lexical items with high frequency are better repeated than these with low frequency.

2. Long non words will be more difficult to repeat than short non words, in both categories of target like and non target like non words. This hypothesis was derived from Schwartz and Girbau study where they found non-words with 3 and more syllables more taxing than shorter ones. Furthermore, Balthazar (2003) claimed that a list of short words like "bat, key, cub, man, duck" is easier to recall than the list of long words like "hamburger, alligator, Halloween, tuition, metaphor." explaining that it is more time consuming to subvocally repeat long words than short ones. Other researchers indicated; the longer the words, the less accurate the recall will be (Engelkamp \& Rummer 1999, Rummer \& Engelkamp, 2001, Willis \& Gathercole 2001). 
Vol. 5, No. $4 \quad$ Jaber-Awida: Experiment in Non Word Repetition by Monolingual...

\section{Method}

Setting

The experiment was conducted in the children's kindergarten. Each child was tested individually in a quiet room.

\section{Subjects}

Twenty preschool children, ages 5 to 6 . They are Arabic monolingual children with no history of speech therapy. The children come from a low to-middle socioeconomic status.

Design

The experiment included 30 non words, ranging from 2 to 4 syllables each. Each category of syllables included 10 non words. Half of the non words were similar to real words (target-like) and half were not similar (non target-like). The words contain one cluster at the most, and this is because we wanted to control for cluster-hood and complexity, since the subjects are young and would struggle with words containing multiple clusters.

In both groups, the target like and non target like I controlled for the number of consonant onset, vowel onset, consonant suffix, vowel suffix, and consonant clusters. In the 2 syllable length all non words began with a consonant onset in both groups; target like and non target like. In the 3 and 4 syllable length I had 3 non words with consonant onset and 2 with vowel onset; in both groups of target like and non target like items in order to have variability. I managed to keep the long items ( 3 and 4 syllables) similar to each other in features more than the category of 2 syllables in order to be able to make a clear distinction between them.

The suffix of target like and non target like non words was also controlled as follows: in the 2 syllable length there were 3 non words ending with a vowel, and 2 non words ending with a consonant. However, similar features were chosen for the items having the length of 3 and 4 syllables: 4 non words ended with a consonant and 1 ended with a vowel in the non target like and target like category.

In addition, I did not include non words with the length of 5 syllables because it was difficult to find words of such length in Arabic, and also 5 year old children are less familiar with words of such length.

The non words used in this study were consistent with patterns of Arabic word structure (i.e. conjugations). Among the Arabic conjugations used in the data were:

faala, iftiaal, infaala, muftaal, and more.

For example: the non word "idkaman" follows the structure of the conjugation "iftiaal". 
$\underline{\text { Items division }}$

$3 \times 2$

\begin{tabular}{|l|l|l|}
\hline Target like/2 syllables & 3 syllables & 4 syllables \\
\hline Non target like & & \\
\hline
\end{tabular}

Procedure

The children were familiar with the experimenter because they already previously participated in another experiment. The experimenter started each child's session following the protocol's instructions, saying:

"Hi, I will show you now some funny words you never heard before, and I will see if you can repeat them.

I will say for example: "kapan" and you will repeat "kapan".

I will say "makfena" and you will repeat "makfena".

Good, I see you understand what you have to do. Let's go on...".

Then the experimenter uttered each stimulus (non word) and listened carefully to the child's repetition. Responses were recorded immediately on a scoring sheet made for each subject.

The experimenter started out by uttering the non target like items, and then the target like ones. At the end of each child's turn; the experimenter rewarded the child with a colorful stick for his/her participation and effort.

\section{Findings}

The results of the non word repetition experiment showed various patterns and a correlation between children's performance and the influence of word likeness and length. Generally, children erred more on long (i.e. 4 syllables) nonwords which were target like and non target like words. Figure 1 illustrates their performance in percentages.

In Figure 1 we see a similar pattern of more errors in the long non words. This finding is consistent with the second hypothesis, which is also similar to the findings of many studies on non words repetition stating that long non words are harder to repeat than short non words (Girbau \& Schwartz 2008, Balthazar 2003, Engelkamp \& Rummer 1999, Rummer \& Engelkamp 2001, Willis \& Gathercole 2001). 
Figure 1. Children'S Rate of Success in Repeating Non Target-Like and Target-Like Non Words



\section{Classification of Errors}

Subjects committed a mixture of errors which were classified into subgroups. The categories of errors found in the data were: (1) syllable omission (2) cluster reduction (3) consonant/vowel omission (4) transpositions (5) substitution (6) substitution+omission (7) transposition+omission/ substitution. Some of the errors were more prevalent than others.

Non target-like non words caused more errors than the target-like non words (134 and 100 respectively). Also the longer the words; the more errors were found. Table 1 presents types of errors for each syllable length.

Table 1. Types of Errors for Each Syllable Length in Non Target-Like Items

\begin{tabular}{|l|c|c|c|c|}
\hline & 2 Syllables & 3 Syllables & 4 Syllables & Total \\
\hline Syllable Omission & 1 & 2 & 12 & 15 \\
\hline Cluster Reduction & 0 & 2 & 0 & 2 \\
\hline Consonant/Vowel Omission & 2 & 0 & 2 & 4 \\
\hline Transpositions & 0 & 2 & 4 & 6 \\
\hline Substitution & 0 & 11 & 19 & 30 \\
\hline Substitution+Omission & 0 & 1 & 7 & 8 \\
\hline Transposition+Ommission/Substitution & 0 & 1 & 1 & 2 \\
\hline
\end{tabular}

We can deduce from the graph in Figure 2 that substitution was prevalent error in children's repetitions (i.e. 30 instances). For example: subject A9 produced the non word "ishradagat" for "ishradajak". Similarly, the most prevalent type of error in Girbau and Schwartz (2008) study was substitution for the control group and the impaired one. Syllable omission was the second most common error among the errors made in the non-target like non words (i.e. 15 instances). For example: subject A17 said "imkarar" instead of "imkadarar", omitting the syllable -da. It is worth noticing that mostly errors occur in the 4 syllable length non words (i.e. 45 errors in the 4 syllable length, 19 errors in the 3 syllable length, and 3 errors in the 2 syllable length). This finding can be supported by Balthazar (2003) experiment in which it was found 
that word length effect plays a role in the repetition of non words. Omission errors might also be a result of a limitation of children's short term memory at this young age of preschool. Thus, preschool children perhaps tended to omit syllables in order to decrease the work-load on their short term memory, because many researchers claim that the longer the words the less accurate their recall will be (Engelkamp \& Rummer 1999, Rummer \& Engelkamp 2001, Willis \& Gathercole 2001). Types of errors for each syllable length for target like items are presented in Table 2, while the amount of errors in each type is presented in Figure 3.

Figure 2. Amount of Errors Children's Committed in Each Category-Non Target Like Items

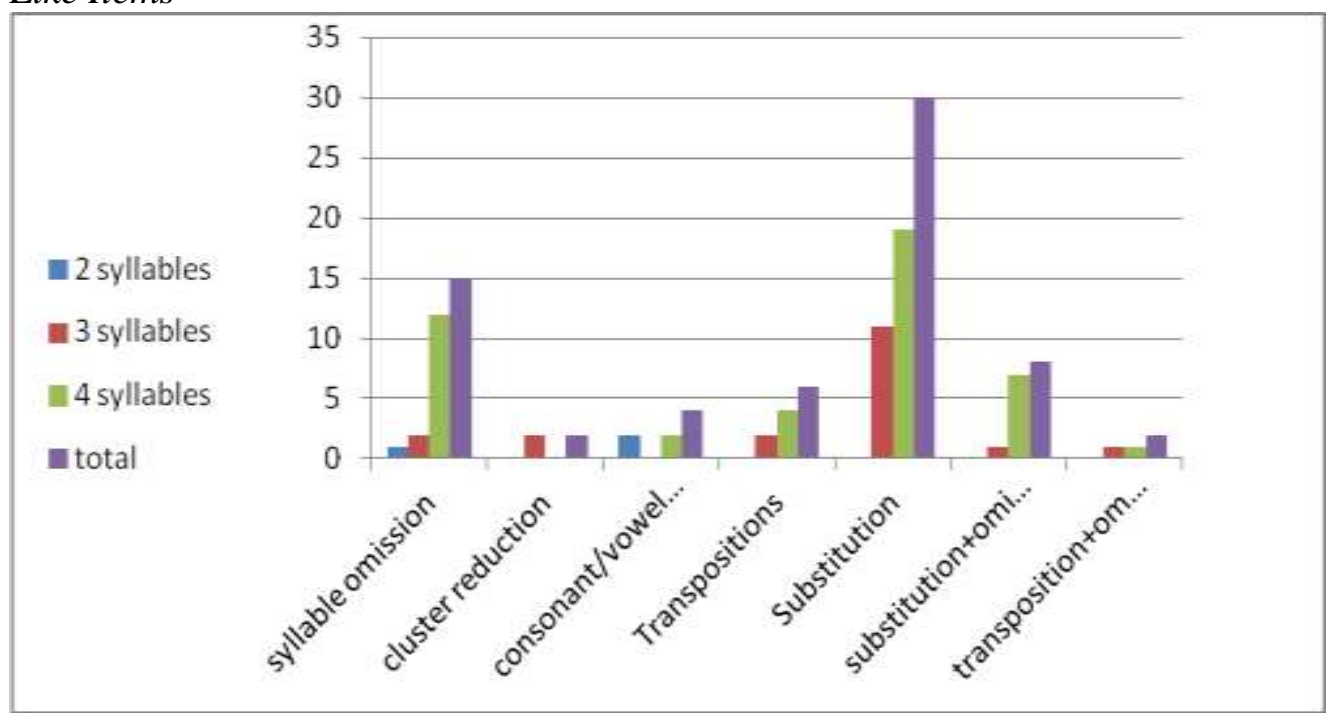

Table 2. Types of Errors for Each Syllable Length in Target-Like Items

\begin{tabular}{|l|c|c|c|c|}
\hline & 2 Syllables & 3 Syllables & 4 Syllables & Total \\
\hline Syllable Omission & 0 & 0 & 3 & 3 \\
\hline Cluster Reduction & 0 & 0 & 0 & 0 \\
\hline Consonant/Vowel Omission & 0 & 0 & 5 & 5 \\
\hline Transpositions & 0 & 1 & 9 & 10 \\
\hline Substitution & 2 & 4 & 16 & 22 \\
\hline Substitution+Omission & 0 & 0 & 9 & 9 \\
\hline Transposition+Ommission/Substitution & 0 & 0 & 1 & 1 \\
\hline
\end{tabular}


Figure 3. Amount of Errors Children's Committed in each Category-Target Like Items

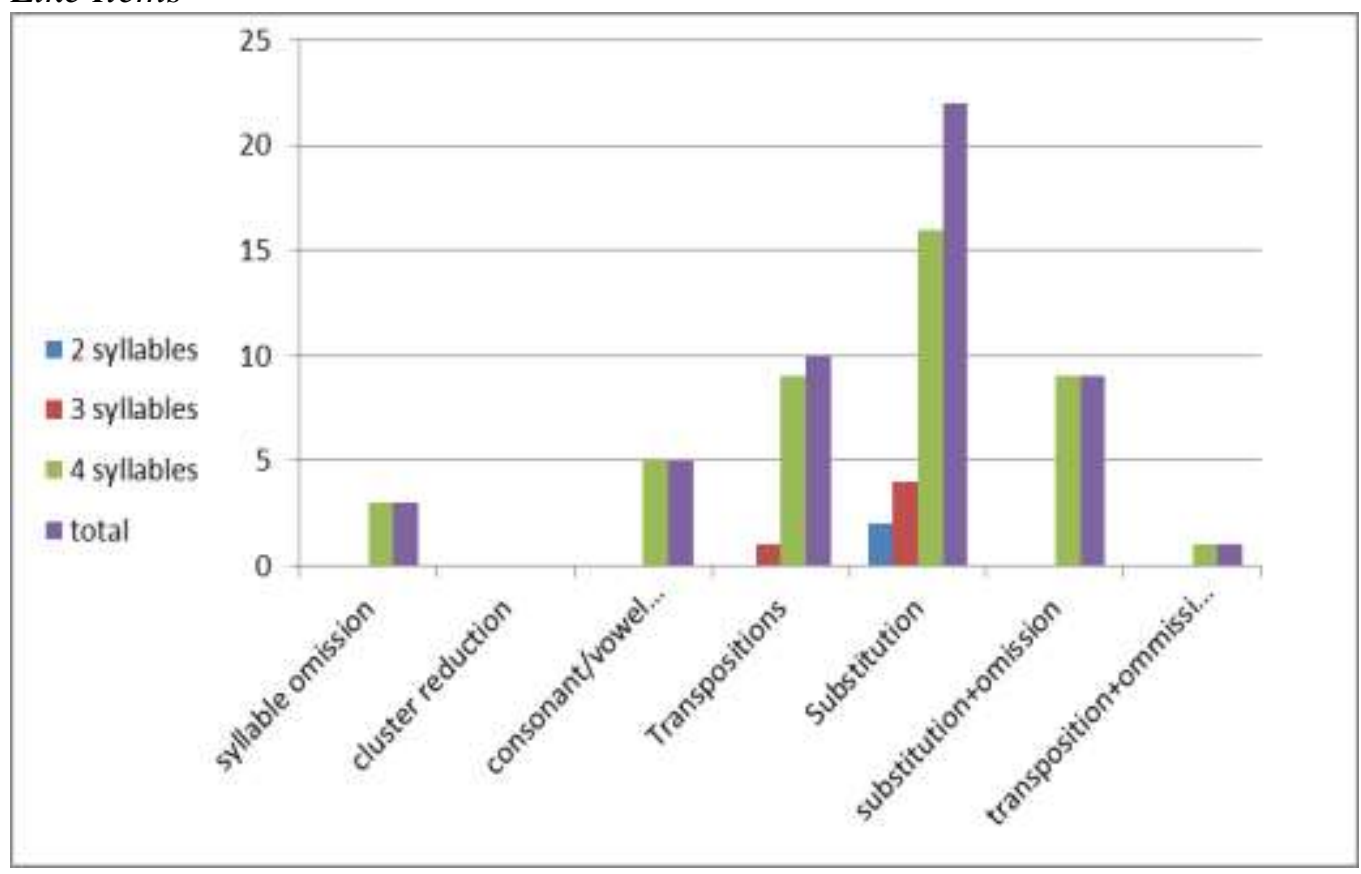

Again, substitution was the more frequent error children committed when repeating non words which are target-like items (i.e. 22 instances). for example: subject A8 produced "malkat" instead of "mardak"; substituting the consonant "r" with "l" and the syllable "dak" with "kat". This follows Girbau and Schwartz (2008) findings of having substitution as the most common error subjects committed. Transpositions were in the second place in terms of errors frequency (i.e. 10 instances). For example, subject A7 produced "mulkabadat" when hearing "mulkadabat", transporting the positions of the consonants "b" and "d". Such types of errors can be explained by Baddeley's argument that "high phonological similarity of words negatively affects the comprehension and recall of words" (1986). Consequently, children made the above mistake due to the high phonological similarity between the two consonants "b" and "d". Once again, syllable length revealed a negative effect on the recall of non words which are target-like as well, triggering 43 errors in the 4 syllable length non words, 5 errors in the 3 syllable length, and 2 errors in the 2 syllable length. This finding follows the pattern found in many other studies such as Balthazar (2003) in which she claimed that a list of short words like "bat, key, cub, man, duck" is easier to recall than the list of long words like "hamburger, alligator, Halloween, tuition, metaphor." explaining that it is more time consuming to subvocally repeat long words than short ones (Balthazar 2003). Other researchers indicated; the longer the words, the less accurate the recall will be (Engelkamp \& Rummer 1999, Rummer \& Engelkamp 2001, Willis \& Gathercole 2001). 


\section{Non Word Likeness}

Non target-like non words caused more errors than the target-like non words (134 and 100 respectively). This finding confirms the first hypothesis as well as many previous studies. For instance, words with high non word likeness were easier to repeat than non words with low word likeness (Coady et al. 2005, Gathercole 1995, Munson et al. 2005).

\section{Individual Children Results}

There were four children which erred more than the rest of the subjects in the experiment session. The children were: A8, A12, A15, and A19. They had more than 10 errors in total for the two groups of non words; target-like and non targetlike. A8 had 11 errors out of 30 (i.e. 11/30), A12 had 14/30, A15 had 12/30, and A19 13/30. The common feature of the mistakes made by the four subjects was that their errors occurred mainly in the long non words, and especially in the non target-like items. I would explain this result by stating Chiat (2006) proposing that in the first year of children's lives they are equipped with sensitivity to the "properties and frequency of rhythmic patterns in phonological input" and they utilize this sensitivity to segmenting words for storage. So my subjects are already 5 years old, and they already have used the mentioned sensitivity, it will be easier for them to use this ability since they are familiar with it from their early years. Accordingly children will vary in their ability to repeat non words because they will differ in their early phonological sensitivity. Thus, these four subjects might be not as developed as the rest of the subjects in terms of phonological sensitivity.

\section{Individual Items which Triggered More Errors}

Some individual items triggered more errors when repeated by children. I chose the items which were repeated erroneously by 10 children and above. The non words were: "mulkadabat" (4 syllables, target-like), and "ibkalamar" (4 syllables, target-like); "ishradajak" (4 syllables, non target-like), and "xusfukiral" (4 syllables, non target-like). Provided are examples of errors made when repeating each of the items listed above:

$$
\begin{aligned}
& \text { mulkadabat } \rightarrow \text { mulkabatat } \\
& \text { ibkalamar } \rightarrow \text { ibkarar } \\
& \text { ishradajak } \rightarrow \text { ishradajad } \\
& \text { xusfukiral } \rightarrow \text { xuskufiral }
\end{aligned}
$$

Interestingly, we can conclude that the high number of errors on these items specifically by the majority of subjects is supported by the many studies claiming that word length negatively influences non word repetition (Girbau \& Schwartz 2008, Balthazar 2003, Engelkamp \& Rummer 1999, Rummer \& Engelkamp 2001, Willis \& Gathercole 2001). 
Vol. 5, No. $4 \quad$ Jaber-Awida: Experiment in Non Word Repetition by Monolingual...

\section{Conclusion and Discussion}

This experiment was led by two hypotheses: (1) it will be easier for preschool children to repeat non words which are target like items, (2) long non words will be more difficult to repeat than short non words, in both categories of target like and non target like non words. Both hypotheses were confirmed by the results of this experiment.

The first hypothesis was derived from studies which confirmed that high word likeness positively influences the performance of children in repeating non words (Coady et al. 2005, Gathercole 1995, Munson et al. 2005). In the conducted experiment, the significant difference was seen in the tri-syllabic non words. The percentage of correctly repeating these non words was $95 \%$ in the target-like items while it was $81 \%$ in the non target-like items.

Yet, it is important to mention that the results of this study would not be representing all the patterns in other populations. The reason behind my claim is that each culture or population has a different degree of exposure or frequency of words; meaning, what might be very frequent in one culture or dialectic area might not be as frequent in the other. Thus, we should take into consideration that the social background of the children might have an effect on the observed results. Future studies which can control for the social or background variable in order to measure frequency of words and equalize among subjects are in need.

The second hypothesis was also confirmed and was supported by numerous studies (e.g. Girbau and Schwartz (2008), Balthazar (2003), Engelkamp and Rummer (1999), Rummer and Engelkamp (2001) and Willis and Gathercole (2001). In all of the mentioned studies it was proven that the longer the non word, the higher the chances are for repetition errors. Results of the conducted experiment showed that: in the target-like items non words with 2 syllable length had 2 error instances, 3 syllable non words had 5 error instances, and 4 syllable non words reached 43 errors. On the other hand, non target-like items resulted in: 3 errors in the 2 syllable non words, 19 errors in the 3 syllable items, and 45 in the 4 syllable non words.

Apparently word length taxed children's memory capacities in this study. The question which remains unanswered is: would this pattern of results be observed also in children who are very language competent, meaning, which have more practice in using long words in their language, or children who have a language which has long words in nature? I believe that this question bears different results than the ones we have in my experiment. Thus, one might claim that the results in this study are language dependent and cannot be generalized or accounted for another language.

The nature of errors made in both groups of non words (i.e. target like and non target like) varied. Cluster reduction was almost not found in the errors of children. I would explain this by claiming that the clusters I embedded in the non words were not high in complexity, thus children managed to repeat them correctly. In addition, the clusters in each non word were centralized (at the middle of the non word), preceded and followed by a vowel, which made it easier to 
subject to repeat them. It is difficult for speakers to produce word initial clusters for example.

Substitution was the most prevalent type of error in both groups of target like and non target like non words ( 22 errors and 30 errors respectively). This is similar to the result Girbau and Schwartz had, where substitution was the most prevalent error their subjects made (both SLI and TD). These researchers explain this result by claiming that the phonological working memory abilities are responsible for this occurence.

Notably, children committed more errors of syllable omission in the non target like words than in the target like words (15 vs. 3 respectively). Many researchers would attribute this kind of error to the effect of word likeness (Coady et al. 2005, Gathercole 1995, Munson et al. 2005). Children simply omitted more syllables in the non target like group because these words are similar to any words in their lexicon so they perhaps tried to omit syllables to make it easier for themselves to process and maybe turn the words into something similar to words in their lexicons.

It is interesting to notice that substitution error was committed more frequently as the length of non words increased. For example: in the 3 syllable length the most prevalent error was substitution (4 in the target like group and 11 in the non target like group). The picture changed when the words consisted of 4 syllables the substitution errors increased in number (16 in the target like group and 19 in the non target like group). Balthazar would explain this by saying that it is more time consuming to subvocally repeat long words than short ones (2003). Likewise, some researchers have commented that the longer the words are the less accurate children would repeat them (e.g. Engelkamp \& Rummer 1999, Rummer \& Engelkamp 2001, Willis \& Gathercole 2001).

In conclusion, since it was indicated that word length and likeness influence non word repetition, it is highly significant to conduct further research on different populations in order to have a wider view of the phenomenon of non word repetition. I would suggest that examining the difference in performance between males and females would perhaps reveal interesting features of the nature of processing in each of the genders. Cross linguistic research on non word repetition under timing conditions would also be very motivating to check if pressure or time constraints would trigger different performance on the part of the subjects.

\section{Acknowledgements}

Special thanks to Professor Sharon Armon-Lotem, who without her supervision and guidance this paper would not have been written.

\section{Bibliography}

Alloway TP, Gathercole SE (2005) The Role of Sentence Recall in Reading and Language Skills of Children with Learning Difficulties. Learning and Individual Differences 15: 271-282. 
Vol. 5, No. $4 \quad$ Jaber-Awida: Experiment in Non Word Repetition by Monolingual...

Archibald LMD, Gathercole S (2006) Nonword Repetition: A Comparison of Tests. Journal of Speech, Language, and Hearing Research 49: 970-983.

Baddeley AD (1986) Working memory. Oxford: Clarendon Press.

Baddeley AD (2000) The Episodic Buffer: A New Component of Working Memory? Trends in Cognitive Sciences 4: 417-423.

Baddeley AD (2003) Working Memory and Language: An Overview. Journal of Communication Disorders 36: 189-208.

Baddeley AD, Vallar G, Wilson BA (1987) Sentence Comprehension and Phonological Memory: Some Neuropsychological Evidence. In M Coltheart (Eds.), Attention and Performance XII: The Psychology of Reading (pp. 509-529). London: Lawrence Erlbaum Associates.

Balthazar CW (2003) The Word Length Effect in Children with Language Impairment. Journal of Communication Disorders 36: 487-505.

Bishop DVM, North T, Donlan C (1996) Non-word Repetition as a Behavioural Marker for Inherited Language Impairment: Evidence from a Twin Study. Journal of Child Psychology and Psychiatry 37: 391-403.

Butterworth B, Shallice T, Watson FL (1990) Short-term Retention without Short-term Memory. In G Vallar, T Shallice (Eds.), Neuropsychological Impairments of Shortterm Memory (pp. 187-213). New York, NY, US: Cambridge University Press.

Chiat S (2006) The Developmental Trajectory of Nonword Repetition. Applied Psycholinguistics 27: 552-556.

Coady JA, Kluender K, Evans JL (2005) Categorical Perception of Speech by Children with Specific Language Impairments. Journal of Speech, Language, and Hearing Research 48(4): 944-959.

Conti-Ramsden G (2003) Processing and Linguistic Markers in Young Children with Specific Language Impairment (SLI). Journal of Speech, Language, and Hearing Research 46: 1029-1037.

Conti-Ramsden G, Botting N, \& Faragher B (2001) Psycholinguistic Markers for Specific Language Impairment. Journal of Child Psychology and Psychiatry 42: $741-748$.

Cuvo AJ (1975) Developmental Differences in Rehearsal and Free Recall. Journal of Experimental Child Psychology 19: 265-278.

Edwards JD, Lahey M (1998) Nonword Repetitions of Children with Specific Language Impairment: Exploration of some Explanations for their Inaccuracies. Applied Psycholinguistics 19: 279-309.

Ellis Weismer S, Tomblin JB, Zhang X, Buckwalter P, Chynoweth JG, Jones M (2000) Nonword Repetition Performance in School-age Children with and without Language Impairment. Journal of Speech, Language, and Hearing Research 43: 865-878.

Engelkamp J, Rummer R (1999) Syntaktische Struktur und Wortlänge im Satzrecall (Syntactic structure and word length in sentence recall). Zeitschrift für experimentelle Psychologie 46: 1-15.

Gathercole SE (2006) Nonword Repetition and Word Learning: The Nature of the Relationship. Applied Psycholinguistics 27(4): 513-543.

Gathercole SE (1995) The Assessment of Phonological Memory Skills in Preschool Children. British Journal of Educational Psychology 65: 155-164.

Gathercole SE, Baddeley AD (1990) Phonological Memory Deficits in Language Disordered Children: Is there a Causal Connection? Journal of Memory and Language 29(3): 336-3.

Gathercole SE, Baddeley AD (1996) The Children's Test of Nonword Repetition. London: The Psychological Corporation UK. 
Gathercole SE, Pickering SJ (2000) Assessment of Working Memory in Six- and Seven-year-old Children. Journal of Educational Psychology 92: 377-390.

Girbau D, Schwartz RG (2008) Phonological Working Memory in Spanish-English Bilingual Children with and without Specific Language Impairment. Journal of Communication Disorders 41(2): 124-145.

Graf Estes K, Evans JL, Else-Quest NM (2007) Differences in the Nonword Repetition Performance of Children with and without Specific Language Impairment: A MetaAnalysis. Journal of Speech, Language, and Hearing Research 50: 177-195.

Gray S (2003) Diagnostic Accuracy and Test-retest Reliability of Nonword Repetition and Digit Span Tasks Administered to Preschool Children with Specific Language Impairment. Journal of Communication Disorders 36 129-151.

Hagen JW, Kail RV (1973) Facilitation and Distraction in Short-term Memory. Child Development 44 831-836.

Hanten G, Martin RC (2000) Contributions of Phonological and Semantic Short-term Memory to Sentence Processing: Evidence from Two Cases of Closed Head Injury in Children. Journal of Memory and Language 43(20): 335-361

Kail R (1990) The Development of Memory in Children (3rd ed.). New York: W.H. Freeman and Company.

Kail R (1994) A Method for Studying the Generalized Slowing Hypothesis in Children with Specific Language Impairment. Journal of Speech and Hearing Research 37: 418-421.

Keeney TJ, Cannizzo SR, Flavell JH (1967) Spontaneous and Induced Verbal Rehearsal in a Recall Task. Child Development 38: 953-966.

Lahey M, Edwards J, Munson B (2001) Is Processing Speed Related to Severity of Language Impairment? Journal of Speech, Language, and Hearing Research 44: 1354-1361.

Martin RC, Breedin SD, Damian MF (1999) The Relation of Phoneme Discrimination, Lexical Access, and Short-term Memory: A Case Study and Interactive Activation Account. Brain Language 70: 437-482.

Montgomery J (1995) Sentence Comprehension in Children with Specific Language Impairment: The Role of Phonological Working Memory. Journal of Speech and Hearing Research 38: 187-199.

Munson B, Kurtz BA, Windsor J (2005) The Influence Of Vocabulary Size, Phonotactic Probability, and Wordlikeness on Nonword Repetitions of Children with and without Specific Language Impairment. Journal of Speech Language and Hearing Research 48: 1033-1047.

Ornstein PA, Naus MJ, Stone BP (1977) Rehearsal Training and Developmental Differences in Memory. Developmental Psychology 13: 15-24.

Rummer R, Engelkamp J (2001) Phonological Information Contributes to Short-term Recall of Auditorily Presented Sentences. Journal of Memory and Language 45: 451467.

Sahlén G (1999) The Impact of Forestry on Dragonfly Diversity in Central Sweden. International Journal of Odonatology 2(2): 177-186.

Sininger YS, Klatzky RL, Kirchner DM (1989) Memory Scanning Speed in Language Disordered Children. Journal of Speech and Hearing Research 32: 289-297.

Van der Lely HKJ, Howard D (1993) Children with Specific Language Impairment: Linguistic Impairment or Short-term Memory Deficit? Journal of Speech \& Hearing Research 36(6): 1193-1207.

Van der Lely HKJ, Marshall C, Marinis T (2007) The Effect of Phonotactics on Passive Comprehension in Typically Developing and Grammatical-SLI Children. Lingua 117: 1434-1447. 
Vol. 5, No. $4 \quad$ Jaber-Awida: Experiment in Non Word Repetition by Monolingual...

Warrington EK, McCarthy R (1987) Categories of Knowledge. Further Fractionations and an Attempted Integration. Brain: A Journal of Neurology 110(5): 1273-1296.

Willis CS, Gathercole SE (2001) Phonological Short-term Memory Contributions to Sentence Processing in Young Children. Memory 9: 349-364. 